\title{
Apparent shape of super-spinning black holes
}

\author{
Cosimo Bambi块 and Katherine Freese 2 † \\ ${ }^{1} I P M U$, University of Tokyo, Kashiwa, Chiba 27ry-8568, Japan \\ ${ }^{2}$ MCTP, University of Michigan, Ann Arbor, Michigan 48109, USA
}

(Dated: November 4, 2018)

\begin{abstract}
We consider the possibility that astrophysical Black Holes (BHs) can violate the Kerr bound; i.e., they can have angular momentum greater than $\mathrm{BH}$ mass, $J>M$. We discuss implications on the $\mathrm{BH}$ apparent shape. Even if the bound is violated by a small amount, the shadow cast by the BH changes significantly (it is $\sim$ an order of magnitude smaller) from the case with $J \leq M$ and can be used as a clear observational signature in the search for super-spinning BHs. We discuss briefly recent observations in the $\mathrm{mm}$ range of the super-massive $\mathrm{BH}$ at the center of the Galaxy, speculating on the possibility that it might violate the Kerr bound.
\end{abstract}

PACS numbers: 97.60.Lf, 95.30.Sf, 04.60.Bc

\section{INTRODUCTION}

Black Holes (BHs) are quite strange objects, which are devoid of a true internal structure and are completely defined by a few parameters [1, 2]. In the case of BHs which we may possibly find in our Universe, the number of these parameters reduces to three: the mass $M$, the charge $Q$, and the spin $J$. In this paper we pay close attention to the possible spin of the $\mathrm{BH}$ while we set $Q=0$. At present good $\mathrm{BH}$ candidates [3] include super-massive objects $\left(10^{5}-10^{9} M_{\odot}\right)$ at the center of galaxies and stellar mass objects $\left(5-20 M_{\odot}\right)$ in X-ray binary systems. In both cases, we can infer their mass from dynamical arguments, studying the Newtonian orbital motion of stars or gas around them ${ }^{1}$. A challenge today is to measure the spin of these objects as may be experimentally feasible in the near future. Here the difficulty is that we need to probe the spacetime close to the horizon, because spin effects are absent in Newtonian gravity and are suppressed at small velocities and large distances.

An important feature is that BHs are expected to respect the Kerr bound $J \leq M$. This is just the condition to have a horizon. If the Kerr bound were violated, instead of a rotating BH we would have a naked singularity. To see this we can examine the 4 dimensional Kerr-Neumann or Reissner-Nördstrom solutions. The position of the horizon is given by the expression [5, [6]

$$
R_{H}=M+\sqrt{M^{2}-Q^{2}-J^{2}}
$$

where $Q$ and $J$ are, respectively, electric charge and angular momentum of $\mathrm{BH}$. It is clear that in $4 \mathrm{D}$ spacetime the horizon cannot be formed if

$$
M<\sqrt{Q^{2}+J^{2}}
$$

In the absence of a horizon, there would be naked singularities which are not allowed. Indeed if condition (2) is fulfilled, the Kerr-Newmann metric makes it possible to reach the physical singularity at $r=0$ from some large $r$ in finite time without crossing any horizon. One could thus consider closed time-like curves and violate causality (see e.g. section 66c of [1] or ref. [7]). For this reason, usually some kind of cosmic censorship is assumed and naked singularities are forbidden [8].

However, it seems reasonable that the singularity at the center of $\mathrm{BHs}$ has actually no physical meaning and it is just the symptom of the breakdown of classical General Relativity. First, it is difficult to believe that all the matter can collapse into an infinitesimal volume. Second, this is usually the kind of pathology which is expected to be solved at the quantum level. On these general grounds, one is tempted to argue that actually there is no singularity at the center and that the Kerr bound may be violated. In particular [9] discusses possible origins of the breach of the Kerr bound in string theory.

\footnotetext{
*E-mail: cosimo.bambi@ipmu.jp

${ }^{\dagger}$ E-mail: ktfreese@umich.edu

${ }^{1}$ It is also possible that a third category of BHs exists, intermediate mass BHs $\left(10^{3}-10^{4} M_{\odot}\right)$, but so far we do not have much information about them and, in particular, there are no clear measurements of their mass [4].
} 
One more comment is in order here. Since for $J>M$ there is no horizon, Robinson's theorem does not hold [10] and thus (at least in classical General Relativity) the super-rotating object might not be described by the Kerr metric. In quantum gravity we simply do not know what happens.

The most promising approach to measure the spin of BHs is often believed to be the study of emission lines (notably the fluorescent iron $\mathrm{K} \alpha$ at $6.4 \mathrm{keV}$ ), where $J$ may be deduced by fitting the shape of the line [11]. The method has some weak points. In particular, one has to assume some emissivity function (usually modeled as a power law in the radius) and that there is no emission inside the Innermost Stable Circular Orbit (ISCO). Relaxing these assumptions, one can find quite different results [12]. Another common approach is the $\mathrm{X}$-ray continuum fitting method, which is also based on the fact that the inner edge of the accretion disk is presumably the ISCO [13, 14, 15]. Here we need to know the distance of the $\mathrm{BH}$ candidate, its mass, and the disk inclination angle, but in a few cases there are sufficiently reliable estimates of these quantities. For our purposes, if we want to test the Kerr bound, both approaches do not look suitable, because there is no clear difference between a Kerr BH near extremality and one which violates the Kerr bound by a small amount: the radius of the ISCO is a continuous function of $J$, while we would like to observe some physical quantity which is discontinuous at $J=M$.

In this work we study the apparent shape of a super-spinning BH and we claim that the observation of its shadow could be used to test the Kerr bound. The shadow of the BH is the non-illuminated area seen by an observer if the $\mathrm{BH}$ is in front of a planar light source. In realistic situations, it is usually unlikely to have a bright source of this kind. Nevertheless, something very similar to the shadow can be observed if the BH is surrounded by an emitting medium (typically the accreting gas) which is optically thin (and this is always possible for enough high frequencies). Here an arbitrarily small violation of the Kerr bound makes the horizon disappear and changes significantly the apparent shape of the $\mathrm{BH}$ : now only the photons reaching the center of the $\mathrm{BH}$ are lost, while all the others, with turning points at finite distances from the center (or at distances larger than some scale coming from new physics), are not captured and can therefore come back to infinity.

\section{KERR BLACK HOLES}

In this section we briefly review the study of the apparent shape of a $\mathrm{BH}$ which respects the Kerr bound $J \leq M$ (for more details, see e.g. section 63 of [1] or refs. [7, 16, 17]. Analogous studies of similar objects can be found in refs. [18, 19, 20] ). As described above, the shape of the $\mathrm{BH}$ is just the boundary of its shadow: if you fire a photon inside the shape, it is swallowed by the $\mathrm{BH}$; if outside, the photon is not captured. The geodesics equation for the radial coordinate $r$ in the Kerr metric in Boyer and Lindquist coordinates for massless particles is

$$
\left(r^{2}+J^{2} \cos ^{2} \theta\right)^{2}\left(\frac{d r}{d \lambda}\right)^{2}=\mathcal{R},
$$

where $\theta$ is the polar angle, $\lambda$ is the affine parameter, and

$$
\begin{aligned}
\mathcal{R} & =E^{2} r^{4}+\left(J^{2} E^{2}-L_{z}^{2}-\mathcal{Q}\right) r^{2}+2 M\left[\left(J E-L_{z}\right)^{2}+\mathcal{Q}\right] r-J^{2} \mathcal{Q}, \\
\mathcal{Q} & =p_{\theta}^{2}+\cos ^{2} \theta\left(\frac{L_{z}^{2}}{\sin ^{2} \theta}-J^{2} E^{2}\right) .
\end{aligned}
$$

Here $E, L_{z}$, and $\mathcal{Q}$ are constants of motion and are, respectively, the energy, the component of the angular momentum parallel to the spin of the $\mathrm{BH}$, and the so called Cartan constant. For our discussion, it is convenient to introduce the variables $\xi=L_{z} / E$ and $\eta=\mathcal{Q} / E^{2}$, which are related to the "celestial coordinates" of an observer at infinity by

$$
\begin{aligned}
& x=\frac{\xi}{\sin \theta_{o b s}}, \\
& y= \pm\left(\eta+J^{2} \cos ^{2} \theta_{o b s}-\xi^{2} \cot ^{2} \theta_{o b s}\right)^{1 / 2}
\end{aligned}
$$

where $\theta_{\text {obs }}$ is the angular coordinate of the observer.

One can think of an effective potential for the photon, which has a barrier with a maximum, goes to negative infinity below $r=r_{h}$, where $r_{h}$ is the horizon, and asymptotes to zero at $r \rightarrow$ infinity. One can see that there are three kinds of photon orbits: $i$ ) capture orbits, in which the photon arrives from infinity with energy larger than the barrier of the effective potential and then crosses into the horizon, ii) scattering orbits, in which the photon arrives from infinity with energy less than the barrier of the effective potential and then comes back to infinity, and iii) unstable orbits of constant radius (at $r=3 M$ for $J=0$, the location of the maximum of the effective potential) which separate the 

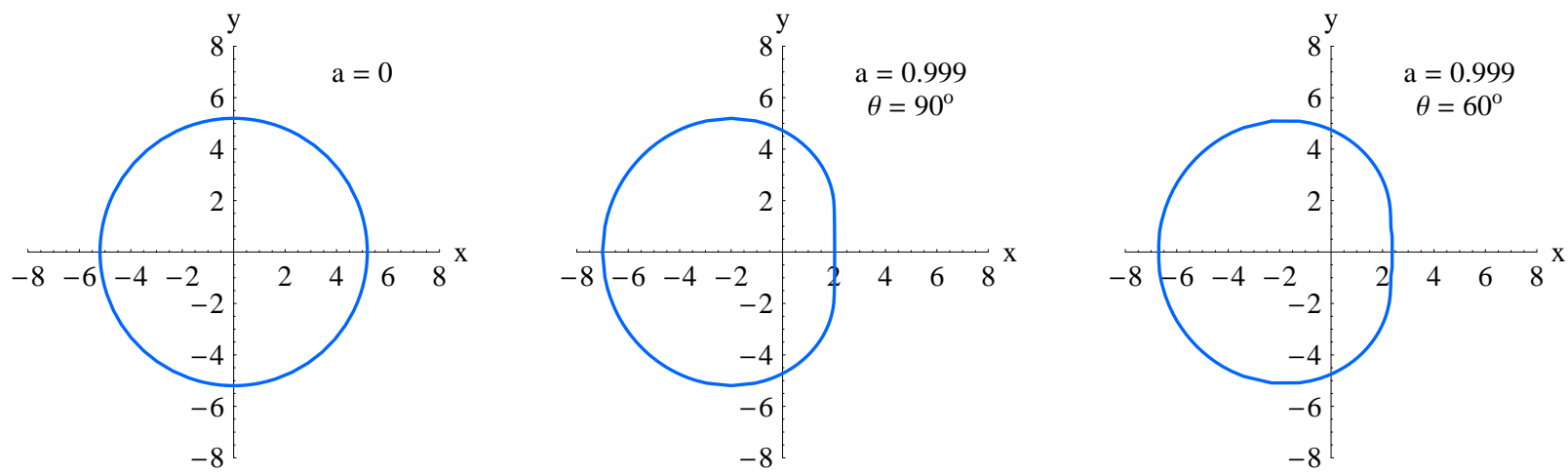

FIG. 1: Apparent shape for Schwarzschild black hole (left panel) and Kerr black hole with $a=J / M=0.999$ (respecting the Kerr bound) for an observer with angular coordinate $\theta_{\text {obs }}=90^{\circ}$ (central panel) and $\theta_{\text {obs }}=60^{\circ}$ (right panel). The unit of length of the coordinate axes is $M$.

capture and the scattering orbits ${ }^{2}$. The apparent shape of the $\mathrm{BH}$ can be found by looking for the unstable orbits. Every orbit can be characterized by the constants of motion $\xi$ and $\eta$, and the set of unstable circular orbits $\left(\xi_{c}, \eta_{c}\right)$ can be used to plot a closed curve in the $x y$-plane which represents the apparent shape of the BH. The latter is larger than the geometrical shape, because the BH bends light rays and thus the actual cross-section is larger than the geometrical one. The equations determining the unstable orbits of constant radius are

$$
\begin{aligned}
\mathcal{R} & =r^{4}+\left(J^{2}-\xi_{c}^{2}-\eta_{c}\right) r^{2}+2 M\left[\left(\xi_{c}-J\right)^{2}+\eta_{c}\right] r-J^{2} \eta_{c}=0, \\
\frac{\partial \mathcal{R}}{\partial r} & =4 r^{3}+2\left(J^{2}-\xi_{c}^{2}-\eta_{c}\right) r+2 M\left[\left(\xi_{c}-J\right)^{2}+\eta_{c}\right]=0 .
\end{aligned}
$$

In the case of Schwarzschild $\mathrm{BH}(J=0)$, the solution is

$$
\eta_{c}\left(\xi_{c}\right)=27 M^{2}-\xi_{c}^{2},
$$

so the apparent image of the BH is a circle of radius $\sqrt{27} M \approx 5.20 M$ (Fig. 1, left panel). In the more general case with $J \neq 0$, one finds

$$
\begin{aligned}
\xi_{c} & =\frac{M\left(r^{2}-J^{2}\right)-r\left(r^{2}-2 M r+J^{2}\right)}{J(r-M)}, \\
\eta_{c} & =\frac{4 J^{2} M r^{3}-r^{4}(r-3 M)^{2}}{J^{2}(r-M)^{2}},
\end{aligned}
$$

where $r$ is the radius of the unstable orbit. The apparent shape of a $\mathrm{BH} J=0.999 M$ is reported in Fig. 1 for an observer on the equatorial plane (central panel) and for one with angular coordinate $\theta_{o b s}=60^{\circ}$ (right panel). The two figures are different, even if it is not very much evident.

The main feature of the shape of rotating BHs is the asymmetry along the spin axis, because of the different effective potential for photons orbiting around the $\mathrm{BH}$ in one or the other direction. The radius of the unstable circular orbit is smaller for photons with angular momentum parallel to the $\mathrm{BH}$ spin and that slightly flattens the $\mathrm{BH}$ shadow on one side. The effect is maximal for the observer on the equatorial plane, $\theta_{o b s}=90^{\circ}$. As $\theta_{o b s} \rightarrow 0^{\circ}$ (or $180^{\circ}$ ), the $\mathrm{BH}$ shape reduces to a circle of radius $\sqrt{\eta_{c}(0)+J^{2}}$, where $\eta_{c}(0)$ is the value of $\eta_{c}$ for $\xi_{c}=0$. One can thus find that the radius of the circle is a little smaller than $\sqrt{27} M$ and decreases as the spin increases. For example, when $J=0.999 M$ the radius is about $4.83 \mathrm{M}$.

\footnotetext{
${ }^{2}$ In the simplest case of $J=0$, the effective potential for massless particles has a maximum at $r=3 M$, the location of the unstable orbit (there is no minimum of this potential). For $J \neq 0$, the picture is qualitatively the same, but a little more complex, because the spin breaks the spherical symmetry of the system. In particular, the effective potential is different for particles with angular momentum parallel or antiparallel to the BH spin.
} 


\section{SUPER-SPINNING BLACK HOLES}

If the $\mathrm{BH}$ violates the Kerr bound and thus has $J>M$, the picture of photon orbits changes. In particular, it is not true that there are unstable orbits of constant radius separating the capture and the scattering trajectories. The apparent shape of the $\mathrm{BH}$ can now be found looking for the set of points $\left(\xi_{s}, \eta_{s}\right)$ for which there is no turning point (and no circular orbits), that is, when eq. (7) has no solution for real and positive $r$. In classical General Relativity, one generally avoids this super-spinning case because (as discussed previously) there is no horizon. Or, one can treat this case by extending the spacetime to include regions with negative value of the radial coordinate $r$. Then when $J>M$, there are photon orbits with a turning point at $r<0$ and thus carry information from "another universe" before coming back to infinity [1].

Here, instead, in a possible extension of GR we believe that it is more reasonable to expect that such photons are captured by the object replacing the singularity. Here we require that the turning point is at $r>0$ (or even at $r>R$, where $R$ is some new distance, see below) because we are assuming that the region of high curvature is modified by quantum effects, but we do not know how. For given $\eta_{s}$, one can solve eq. (7) to find $\xi_{s}$ as a function of $r$

$$
\xi_{s}=\frac{2 J M r \pm \sqrt{4 J^{2} M^{2} r^{2}-\left[r^{4}+\left(J^{2}-\eta_{s}\right) r^{2}+2 M\left(J^{2}+\eta_{s}\right) r-J^{2} \eta_{s}\right]\left(4 M r-r^{2}\right)}}{2 M r-r^{2}} .
$$

For $\eta_{s}=0$, there are no solutions for $\xi_{s}$ in the interval $(-(6 \cosh \chi+\cosh 3 \chi) M, J)$, where $\cosh 3 \chi=J / M$, for any $r>0$. On the other hand, for $\eta_{s}>0, \xi_{s}$ can have any value. The apparent image of a $\mathrm{BH}$ with $J=1.001 M$ for an observer on the equatorial plane is shown in Fig. 2, left panel. Since we are assuming that classical General Relativity breaks down (our basic ingredient to consider the possibility of violation of the Kerr bound), but we do not know what the spacetime near the former singularity could be, we may make the following proposal. One may imagine that quantum gravity effects replace the singularity with something larger, say a core of radius $R$; we then demand that the radius of the turning point of photon orbits is larger than this distance $R$. We may require that only photons with turning point at $r>R$ can really come back to infinity and be detected by the observer. In this case, as $R$ increases, the photon capture cross-section would also increase. Fig. 2 shows the case $R=0$ (left panel), $R=0.01 M$ (central panel), and $R=0.10 M$ (right panel). Even if such a proposal could sound crazy in standard General Relativity, it is likely the simplest way to parametrize new physics.

Quantum gravity effects are presumably important in the region where the spacetime curvature approaches the Planck scale; i.e., very close to the center of the BH. Thus the radius $R$ is likely to be very small, i.e., close to 0 . The case $R=0.01 M$ is still conservative, since the curvature of the spacetime for astrophysical BHs is still tiny (in Planck units) at the distance $0.01 M$ from the center. The shape of the BH (the cross section for capture) is still very small. The purpose of considering several values of $R$ is just to show some (reasonable?) alternatives. Moreover, we do not know if a sphere is the best choice for the shape of this boundary, especially given the extreme rotation of the object. For example, an oblate spheroid could be a reasonable possibility. For an oblate spheroid, the apparent shape of the $\mathrm{BH}$ would be something in between the two spherical cases with $R$ equal, respectively, to the length of the major and minor axes. However, probably only a significant deviation from spherical symmetry could be distinguished observationally.

Let us now study the BH shape for an observer not on the equatorial plane. Using eq. (6), it is easy to see how the shape changes. The case $\theta_{o b s}=60^{\circ}$ is reported in Fig. 3. The shape in the left panel is for the case of photons which can have a turning point arbitrarily close to the origin $r=0$. The photons inside the curves have $\eta_{s}<0$ and cannot be seen by observers on the equatorial plane (indeed eq. (6) would provide imaginary value of $y$ ). The apparent shape of the $\mathrm{BH}$ is an ellipse with semiaxis along the $x$ direction equal to $J$ and semiaxis along the $y$ direction equal to $J\left|\cos \theta_{o b s}\right|$. For $\theta_{o b s} \rightarrow 0^{\circ}$ (or $180^{\circ}$ ), the system looks spherically symmetric and the ellipse reduces to a circle of radius $J$.

If we demand that only photons with a turning point at radii larger than some finite value $R$ can come back to infinity, the $\mathrm{BH}$ shadow for observers with $\theta_{o b s} \neq 90^{\circ}$ is of the kind shown in the central and right panels of Fig. 3 . As expected, the shape is larger than the case with $R=0$, but still much smaller than the one of BHs respecting the Kerr bound. For observers with angular coordinate $\theta_{o b s}=0^{\circ}$ or $180^{\circ}$, the $\mathrm{BH}$ shadow reduces to a circle of radius $\sqrt{\eta_{s}(0)+J^{2}}$. For $R=0.01 M$ and $R=0.10 M$, one finds respectively $1.018 M$ and $1.123 M$.

\section{OBSERVATIONAL CONSEQUENCES}

Quantum gravity effects may resolve BH singularities and in the process allow for violations of the Kerr bound, i.e., allow for super-spinning $\mathrm{BH}$ with $J>M$. We have shown that a $\mathrm{BH}$ violating the Kerr bound has no event horizon and has a very different apparent shape, i.e. cross section for capturing photons. Yet corrections to the 

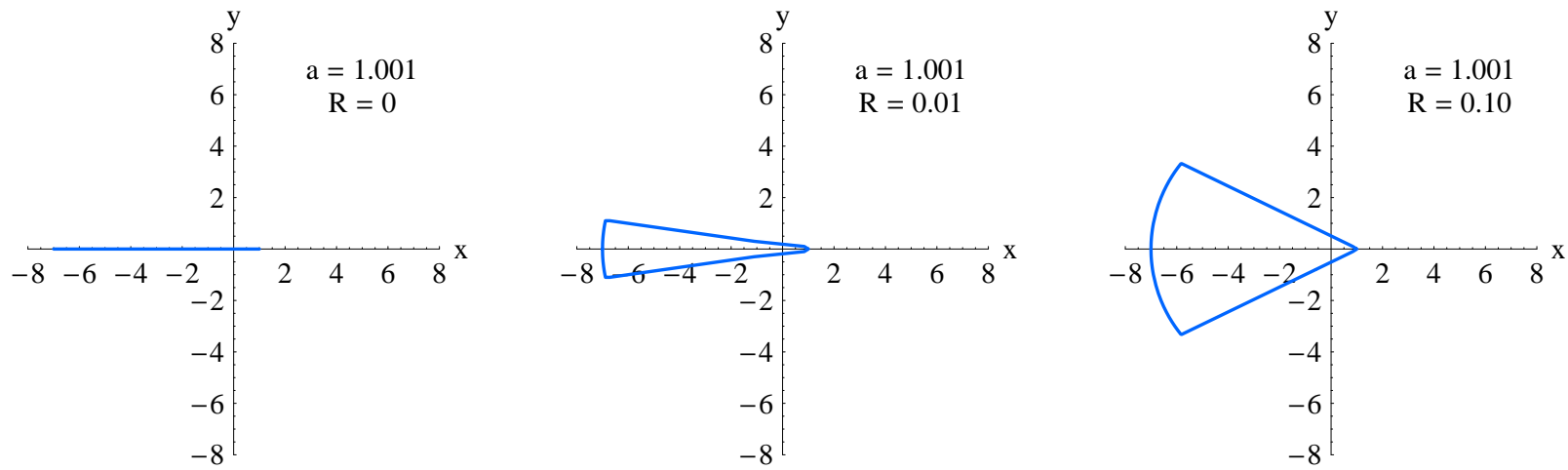

FIG. 2: Apparent shape of a black hole with $a=J / M=1.001$ for an observer on the equatorial plane. Here we demand that the distance of the turning point of photon orbit from the center is larger than 0 (left panel), 0.01 (central panel), and 0.10 (right panel). The unit of length of the coordinate axes is $M$.

spacetime structure are likely to be negligible for astrophysical BHs, because the curvature approaches the Planck scale only in the very central region. We would like to stress that super-spinning BHs cannot be created by spinning up BHs with $J<M$ : on the basis of the Third Law of BH thermodynamics, there are no physical processes capable of transforming a $\mathrm{BH}$ with $J<M$ into an extremal one in a finite number of steps. So, super-spinning BHs must be born as super-spinning BHs. That would violate the cosmic censorship conjecture, but there are some known examples which look physically reasonable and where it is indeed possible to create a naked singularity from the gravitational collapse of matter, see e.g. section 5.7.1 of [2] and references therein. For example, in $2+1$ dimensions where the study of gravitational collapse is more tractable, recent results show that, under general initial conditions, the collapse of a shell with pressure can form a naked singularity and that, in general, angular momentum does not prevent the violation of the cosmic censorship conjecture [21].

The possibility of testing the Kerr bound $J \leq M$ is particularly intriguing for two important reasons. First, even though there are not yet reliable measurements of the BH spin, several indications suggest that astrophysical BHs typically have high spins. For example, in ref. [22] the authors find $J / M>0.93$ for the AGN MCG-6-30-15, while the authors of ref. 23] suggest $J / M>0.8-0.9$ for the stellar mass BH in the X-ray source GX 339-4. Based on the X-ray continuum fitting method, the lower limit on $J / M$ of the BH candidate in the X-ray source GSR $1915+105$ has been estimated to be 0.98 in ref. [14]. The second important point is that observations in the $\mathrm{mm}$ range are now reaching resolutions smaller than the expected angular size of the $\mathrm{BH}$ at the center of the Galaxy [24]. Another promising candidate is the super-massive BH in the center of the galaxy M87, which is about 2000 times more distant, but 1000 times more massive; thus its expected angular size is only a factor 0.5 smaller than the one of the $\mathrm{BH}$ in our own Galaxy.

If a $\mathrm{BH}$ is in front of a light planar source, a distant observer sees its shadow, a non-illuminated area with boundary equal to the $\mathrm{BH}$ apparent shape. However, the region blocked by the $\mathrm{BH}$ is not likely to be completely dark. The $\mathrm{BH}$ is likely to be accreting from a disk which emits radiation itself. Hence, the part of the disk in front of the $\mathrm{BH}$ prevents the BH region from looking completely black; instead, if one is looking in the direction of the $\mathrm{BH}$, one is likely to see a region of reduced illumination rather than a completely dark one. The observer can then see a less illuminated area which has the same form of the shadow. The boundary of this area is not as well defined as the one of the shadow: particles tend to pile up near the last stable orbits. Particles sink to the BH because they lose angular momentum and thus their barrier decreases. This is not a fast process and so matter accumulates around stable orbits and there is the possibility of relevant photon emission, which can somehow compensate the attenuation due to photon redshift.

The rotation of the accreting matter which emits radiation introduces an additional source of uncertainty, deforming this darker image in a way that Schwarzschild or slow rotating BHs may be interpreted as BHs with higher spin value. In the case of super-spinning BHs, basically all the emitted photons can reach the observer at infinity, at least in principle. Photons which are emitted at distances of order $M$ are strongly redshifted if the quantity $J / M$ is slightly larger than one, but at the same time they presumably orbit around the $\mathrm{BH}$ several times, since the orbits are stable, thus increasing the intensity of the flux.

The possibility of observing the shape of the $\mathrm{BH}$ at the center of the Galaxy was first discussed in 25]. The shape should in principle be observable at sub-millimeter wavelengths. Yet, if the spin is below the Kerr bound, measurements of the shape will not definitively determine the spin. The size of the BH shadow turns out to be roughly $10 M$, regardless of the value of the spin, and even if the accretion gas were optically thick and geometrically 

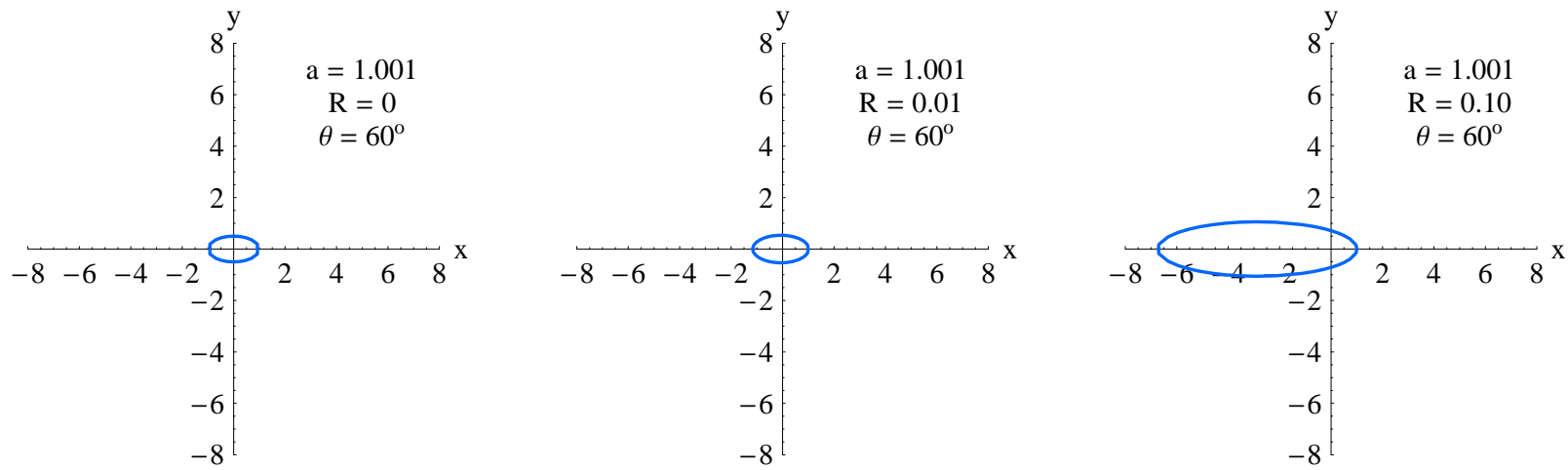

FIG. 3: Apparent shape of a black hole with $a=J / M=1.001$. Here we demand that the distance of the turning point of photon orbit from the center is larger than 0 (left panel), 0.01 (central panel), and 0.10 (right panel). The unit of length of the coordinate axes is $M$.

thin [26]. The measurement of $J$ may instead be achievable through more sophisticated multi-wavelength studies of the $\mathrm{BH}$ image and of its polarization [27]. Although a precise determination of the spin may be difficult, it may be much easier to distinguish whether the spin is above or below the Kerr bound. If the BH violates the Kerr bound, the apparent shape of the $\mathrm{BH}$ would be much smaller than $10 \mathrm{M}$, for any angular coordinate of the observer. For example, if $\theta_{o b s}=0^{\circ}$ or $180^{\circ}$, the shadow of a Kerr BH is a circle of radius in the range $5.20-4.83 \mathrm{M}$; whereas a $\mathrm{BH}$ slightly in violation of the Kerr bound $(J / M$ a little larger than one) has a shadow which is a circle of radius about $1 M$.

We conclude this section with a speculation on the possible value for the spin of the $\mathrm{BH}$ at the center of our Galaxy. In ref. [24], the authors reported the observation at the wavelength of $1.3 \mathrm{~mm}$ of the radio source Sgr A*, which is coincident with the position of the $\mathrm{BH}$ candidate at the level of 10 mas [28]. In fact it is not clear whether the radio source is exactly centered on the $\mathrm{BH}$ [29] or somewhat shifted away from it [30]. Modelling Sgr $\mathrm{A}^{*}$ as a circular Gaussian brightness distribution, the authors of ref. 24] find that the intrinsic diameter of the radio source is $37_{-10}^{+16} \mu$ as at $3 \sigma$. However, in classical GR, if Sgr A* were a spherically symmetric photosphere centered on the $\mathrm{BH}$, one would expect a much larger diameter: the minimum apparent diameter would range from $10.4 M$ corresponding to $52 \mu$ as for a non-spinning BH $(J=0)$, to $9 M$ corresponding to $45 \mu$ as for a BH spinning at the Kerr bound $(J=M)$ and $\theta_{\text {obs }}=90^{\circ}$. Although the current data are not yet capable of absolute confirmation of such a measurement, its implications would be interesting. One possibility is that the radio source is not perfectly centered at the BH. A second possibility is that the radio emission region is indeed a photosphere centered on the $\mathrm{BH}$, but the $\mathrm{BH}$ violates the Kerr bound and thus the emission region may have a small apparent size as discussed in this paper, even smaller than $45 \mu$ as. Independent measurements of the spin of the $\mathrm{BH}$ candidate in the Galactic Center could be possible in the near future, for example, by observing signatures of time variable structure [31]. Here the idea is that the observed periodicity is due to hot spots orbiting the $\mathrm{BH}$ at a few gravitational radii: if that is correct, the fastest one could be associated with the orbital period at the ISCO, which depends on the BH spin and is much shorter for a fast-rotating Kerr BH than for a Schwarzschild BH.

Because of its very low quiescent luminosity in the near IR, it has been argued that the BH candidate in the Galactic Center cannot be an object with a hard surface and must have an event horizon [32, 33]. Our proposal may be an alternative possibility: an object with neither a solid surface like a star nor an event horizon like a true BH.

\section{SUMMARY AND CONCLUSIONS}

In classical General Relativity, BH spinning more rapidly than the Kerr bound, i.e., spinning with $J>M$, would imply the existence of a naked singularity and the violation of causality. However, if quantum gravity effects can resolve the singularity, causality can be restored and we do not need the Kerr bound. Then super-spinning black holes may exist.

In this paper we have discussed how we may observationally identify a black hole which violates the Kerr bound. The key point is the absence of the horizon, which leads to a very different apparent shape for the black hole. By shape we mean essentially the cross section for capturing photons. If the black hole is surrounded by accreting gas which is optically thin, as we believe is the case for the black hole at the center of the Galaxy for sub-millimeter wavelengths, we can presumably see something similar to the black hole shadow. For the standard $J \leq M$, the precise 
measurement of the black hole spin is difficult because the image size is always about $10 M$. On the other hand, the observational difference between $\mathrm{BH}$ with $J<M$ and with $J>M$ should be quite dramatic. The test of the Kerr bound can instead be relatively easy, because we have just to be able to distinguish an image of apparent size about $10 M$ (for the case where the Kerr bound is satisfied) from one of about $2 M$ (where the Kerr bound is violated). A more detailed study of the picture is necessary; in particular, the black hole image has to be evaluated in a more realistic framework, assuming some astrophysical model for the emitting region surrounding the black hole. This will be the subject of another work.

The possible violation of the Kerr bound does not strictly imply the breakdown of classical General Relativity, since the theory does not require $J \leq M$, but is surely something which would be unexpected in the standard framework and which demands new physics.

\section{Acknowledgments}

We would like to thank David Garfinkle for useful comments. C.B. was supported by World Premier International Research Center Initiative (WPI Initiative), MEXT, Japan. K.F. was supported in part by the MCTP and DOE under grant DOE-FG02-95ER40899.

[1] S. Chandrasekhar, The Mathematical Theory of Black Holes (Clarendon Press, Oxford, UK, 1983).

[2] V. P. Frolov and I. D. Novikov, Black Hole Physics: Basic Concepts and New Developments (Kluwer Academic, Dordrecht, Netherlands, 1998).

[3] R. Narayan, New J. Phys. 7, 199 (2005) arXiv:gr-qc/0506078.

[4] M. Coleman Miller and E. J. M. Colbert, Int. J. Mod. Phys. D 13, 1 (2004) arXiv:astro-ph/0308402.

[5] Ch. W. Misner, K. S. Thorn and J. A. Wheeler, Gravitation (W. H. Freeman and Company, San Francisco, California, 1973).

[6] A. Lightman, W. H. Press, R. H. Price and S. A. Teukolsky, Problem Book in Relativity and Gravitation, (Princeton University Press, Princeton, New Jersey, 1975).

[7] B. Carter, Phys. Rev. 174, 1559 (1968).

[8] R. Penrose, Riv. Nuovo Cim. 1, 252 (1969) [Gen. Rel. Grav. 34, 1141 (2002)].

[9] E. G. Gimon and P. Horava, arXiv:0706.2873 [hep-th].

[10] D. C. Robinson, Phys. Rev. Lett. 34, 905 (1975).

[11] C. S. Reynolds and M. A. Nowak, Phys. Rept. 377, 389 (2003) arXiv:astro-ph/0212065.

[12] C. S. Reynolds and M. C. Begelman, Astrophys. J. 488, 109 (1997) arXiv:astro-ph/9705136.

[13] R. Shafee, J. E. McClintock, R. Narayan, S. W. Davis, L. X. Li and R. A. Remillard, Astrophys. J. 636, L113 (2006) arXiv:astro-ph/0508302.

[14] J. E. McClintock, R. Shafee, R. Narayan, R. A. Remillard, S. W. Davis and L. X. Li, Astrophys. J. 652, 518 (2006) arXiv:astro-ph/0606076.

[15] J. Liu, J. McClintock, R. Narayan, S. Davis and J. Orosz, Astrophys. J. 679, L37 (2008) arXiv:0803.1834 [astro-ph].

[16] A. F. Zakharov, F. De Paolis, G. Ingrosso and A. A. Nucita, New Astron. 10, 479 (2005) arXiv:astro-ph/0505286.

[17] V. Bozza, F. De Luca, G. Scarpetta and M. Sereno, Phys. Rev. D 72, 083003 (2005) arXiv:gr-qc/0507137.

[18] K. S. Virbhadra and G. F. R. Ellis, Phys. Rev. D 65, 103004 (2002).

[19] K. S. Virbhadra and C. R. Keeton, Phys. Rev. D 77, 124014 (2008) arXiv:0710.2333 [gr-qc]].

[20] K. Hioki and U. Miyamoto, Phys. Rev. D 78, 044007 (2008) arXiv:0805.3146 [gr-qc]].

[21] R. B. Mann, J. J. Oh and M. I. Park, arXiv:0812.2297 [hep-th].

[22] C. S. Reynolds, L. W. Brenneman and D. Garofalo, Astrophys. Space Sci. 300, 71 (2005) arXiv:astro-ph/0410116.

[23] J. M. Miller et al., Astrophys. J. 606, L131 (2004) arXiv:astro-ph/0312033.

[24] S. Doeleman et al., Nature 455, 78 (2008) arXiv:0809.2442 [astro-ph]].

[25] H. Falcke, F. Melia and E. Agol, Astrophys. J. 528, L13 (2000) arXiv:astro-ph/9912263.

[26] R. Takahashi, J. Korean Phys. Soc. 45, S1808 (2004) [Astrophys. J. 611, 996 (2004)] arXiv:astro-ph/0405099.

[27] A. E. Broderick and A. Loeb, Astrophys. J. 636, L109 (2006) arXiv:astro-ph/0508386.

[28] A. M. Ghez, S. Salim, S. D. Hornstein, A. Tanner, M. Morris, E. E. Becklin and G. Duchene, Astrophys. J. 620, 744 (2005) arXiv:astro-ph/0306130.

[29] R. Narayan, I. Yi and R. Mahadevan, Nature 374, 623 (1995).

[30] H. Falcke and S. Markoff, Astron. Astrophys. 362, 113 (2000) arXiv:astro-ph/0102186.

[31] S. S. Doeleman, V. L. Fish, A. E. Broderick, A. Loeb and A. E. E. Rogers, arXiv:0809.3424 [astro-ph].

[32] A. E. Broderick and R. Narayan, Astrophys. J. 638, L21 (2006) arXiv:astro-ph/0512211.

[33] A. E. Broderick and R. Narayan, Class. Quant. Grav. 24, 659 (2007) arXiv:gr-qc/0701154]. 